\title{
Percepção da sobrecarga familiar nos cuidados ao paciente psiquiátrico crônico
}

Recebido em: 27/07/2011

Aceito em: 16/11/2011

Mohema Duarte de Oliveira ${ }^{1}$

Mariana Ferreira de Sá ${ }^{2}$

Maria Luciene Rocha ${ }^{3}$

Objetivos: avaliar o impacto da sobrecarga familiar nos cuidados ao paciente psiquiátrico crônico. Metodologia: pesquisa bibliográfica por meio de referências publicadas em documentos, livros, revistas, artigos científicos, internet, ou seja, aborda tudo o que foi publicado sobre o tema proposto. Resultados: a convivência com a pessoa acometida por uma doença mental é desgastante para o familiar e, muitas vezes, há dificuldade de compreensão dos sintomas apresentados pelo doente. Conclusão: há a necessidade de desenvolver programas de informação, orientação e apoio aos familiares dos pacientes psiquiátricos.

Descritores: Sobrecarga, Familiares Cuidadores, Pacientes Psiquiátricos.

\section{Perception of the familiar overload in the cares to the chronic psychiatric patient}

Aims: to assess the impact of family burden in caring for chronic psychiatric patients. Methodology: literature search by means of references in published papers, books, magazines, papers, and internet, that is, it approaches all publishings on the theme. Results: living with the person affected by mental illness is stressful for the family and sometimes there is difficulty in understanding the symptoms presented by the patient. Conclusion: there is a need to develop information, guidance and support to families of psychiatric patients.

Descriptors: Overload, Family Caregivers, Psychiatric Patients.

\section{La percepción de la carga familiar en el cuidado de pacientes psiquiátricos crónicos}

Objectivos: Evaluar el impacto de la carga familiar en el cuidado de pacientes psiquiátricos crónicos. Metodología: la literatura a través de referencias de artículos publicados, libros, revistas, periódicos, internet, o las direcciones de lo que se ha publicado sobre el tema. Resultados: la convivencia con la persona afectada por una enfermedad mental es muy estresante para la familia y con frecuencia hay dificultad en la comprensión de los síntomas que presenta el paciente. Conclusión: Hay una necesidad de desarrollar programas de información, orientación y apoyo a las familias de los pacientes psiquiátricos.

Descriptores: Sobrecarga, Los Cuidadores Familiares, Los Pacientes Psiquiátricos.

\section{INTRODUÇÃO}

$\mathrm{N}$ este artigo está sendo apresentado o conhecimento relacionado à sobrecarga vivenciada pelos cuidadores familiares de pessoas com distúrbios mentais crônicos.

A partir da vivência da sobrecarga houve a necessidade de explorar seu significado, os efeitos negativos das incapacidades geradas pelo distúrbio e o papel assumido pelo cuidador familiar.

Atébem poucotempo,aassistênciaaos pacientes psiquiátricos encontrava-se focalizada nos hospitais psiquiátricos, no modelo hospitalocêntrico, cuja atenção restringia-se à internação e medicalização dos sintomas demonstrados pelo paciente, excluindo-o dos vínculos familiares e sociais.

O que se espera da reforma psiquiátrica não é simplesmente a transferência do doente mental para fora dos muros do hospital, "confinando-o" à vida em casa, aos cuidados de quem puder assisti-lo, ou entregue à própria sorte. Espera-se muito mais, incluindo o resgate ou o estabelecimento da cidadania do doente mental, o respeito à sua singularidade e subjetividade, tornando-o sujeito de seu próprio tratamento, sem a ideia de cura como o único horizonte. Espera-se, assim, a autonomia e a reintegração do sujeito à família e à sociedade ${ }^{(1)}$.

A sobrecarga objetiva engloba os sintomas e comportamentos de pacientes psiquiátricos dentro de seus ambientes sociais e suas consequências, enquanto a sobrecarga subjetiva está relacionada às consequências psicológicas para a família(2).

Estudos recentes mostram que, em contraste com os paradigmas anteriores, as famílias de pessoas portadoras de transtornos psíquicos não são hoje percebidas nem como causas, nem como canais instrumentais para a cura do paciente - pois as mesmas não causam os transtornos e também não podem curá-los -, mas como fortes aliados no enfrentamento de situações altamente adversas ${ }^{(3-4)}$.

Um dos efeitos do processo histórico de desinstitucionalização foi a preocupação sobre seu impacto nas famílias, contribuindo 
para que elas adotem ou não as propostas e ações de transformação do modelo de atenção em saúde mental. Essa preocupação impulsionou também, nas últimas décadas, estudos sobre as consequências das doenças psiquiátricas para a família, também chamadas de sobrecarga familiar (family burden), com o objetivo de compreender o papel da família na rehospitalização do paciente no atual contexto dos tratamentos comunitários $^{(5)}$.

Um dos principais efeitos da reforma psiquiátrica, ou seja, a desinstitucionalização, foi a sobrecarga e o impacto na família do paciente. É fácil perceber que a domicialização da doença traz consigo uma desorganização nos hábitos cotidianos. A família não consegue lidar com as mudanças de comportamento do familiar e, acima de tudo, não possui esclarecimento e informações que forneçam respostas objetivas sobre a causa e o prognóstico da doença.

A sobrecarga proveniente das dificuldades decorrentes da baixa renda das famílias não as deixa suportar o convívio com a psicose, tanto por fatores de ordem emocional, como também por motivos financeiros, devido ao fato de terem que prover as necessidades de um adulto improdutivo e carente de cuidados especiais $^{(6)}$.

Tornar-se cuidador de um paciente psiquiátrico requer que os familiares coloquem suas necessidades e desejos em segundo plano(7).

A sobrecarga familiar pode ser definida como sendo o estresse emocional e econômico a que as famílias se submetem quando um parente recebe alta de um hospital psiquiátrico e retorna ao lar, principalmente quando laços familiares foram rompidos pelo padrão de cronicidade, no caso dos pacientes que permanecem por longos períodos em instituições psiquiátricas ${ }^{(8)}$.

A instituição familiar não se mostra favorável no que diz respeito à desinstitucionalização do doente mental, exercendo pressão para que a instituição psiquiátrica continue "a manter a custódia dos pacientes porque esse pesado encargo não é aceito passivamente por ela"(9).

A sobrecarga tem sido definida em dois aspectos: objetivo e subjetivo. A sobrecarga objetiva se refere às consequências negativas concretas e observáveis, resultantes da existência da doença mental na família como perturbações na rotina, na vida social e profissional dos familiares, perdas financeiras, tarefas cotidianas de cuidados com o paciente (higiene, transporte, medicação, alimentação, atividades ocupacionais etc.), supervisão dos comportamentos problemáticos dos pacientes (comportamentos embaraçosos, agressões físicas e verbais, condutas sexuais inadequadas etc.) e o suporte dado ao paciente ${ }^{(7,10-13)}$.

Os cuidadores que se responsabilizam pelos pacientes mais fragilizados investem bastante tempo e energia na busca de tratamento e nas investidas para que eles o aceitem. A interação com os serviços de saúde mental aparecem também como fonte de sobrecarga pois, em boa parte, os contatos são vivenciados como uma experiência frustrante, constrangedora e confusa.

A intensidade da sobrecarga dos familiares tem sido relacionada com algumas variáveis, tais como: características dos pacientes (diagnóstico, intensidade dos sintomas, grau de dependência, idade, sexo, duração da doença e número de hospitalizações), parentesco e frequência de contato entre cuidador e paciente, e características dos cuidadores (sexo, idade, escolaridade e nível socioeconômico) $)^{(7,14-15)}$. Influem ainda na sobrecarga a disponibilidade de redes de suporte social, as leis sócio-sanitárias, a estrutura e a adequação dos serviços de saúde mental.

\section{METODOLOGIA}

Para nos aproximarmos do objeto de estudo, propomo-nos a realizar uma revisão bibliográfica sobre a percepção da sobrecarga familiar nos cuidados ao paciente psiquiátrico crônico. Houve uma consulta a vários trabalhos científicos que foram classificados em: artigos de pesquisa; artigos teóricos e/ ou de revisão de literatura; monografias; trabalhos apresentados em congressos; teses e dissertações.

Este estudo foi estruturado de acordo com a metodologia da ABNT. Definido o tema da pesquisa, partiu-se para o levantamento que foi realizado por meio da busca eletrônica, nas bases de dados digitais da Scielo (Scientifc Eletronic Library Online) e outros sites sobre o assunto. Procuramos vários estudos relacionados ao tema de pesquisa, porém, não estabelecemos nenhum limite, pois o objetivo era localizar diferentes estudos independentemente do critério.

De posse das informações iniciou-se a leitura e a "triagem" dos textos, ou seja, partiu-se para a análise e a interpretação do material de acordo com o tema escolhido. Após este ter sido organizado e categorizado em áreas temáticas, iniciou-se a redação, culminando dessa forma com o ciclo da pesquisa de revisão bibliográfica.

\section{RESULTADOS E DISCUSSÃO}

A literatura da área indica as mães como as principais cuidadoras dos pacientes e que elas sentem mais sobrecarga do que os demais familiares ${ }^{(13)}$.

Devido à alta frequência, as tarefas cotidianas de assistência aos pacientes acarretaram elevada sobrecarga objetiva para a maioria dos cuidadores. A necessidade de realizar frequentemente tais tarefas levou à constatação de que as restrições do lazer e de atividades sociais são as principais alterações permanentes na vida dos cuidadores e que tais restrições geram elevada sobrecarga nesses familiares ${ }^{(16-20)}$.

Além disso, os familiares se preocupavam frequentemente com o futuro dos pacientes, em especial sobre como seriam suas vidas após a morte dos cuidadores atuais. Tornar-se cuidador de um paciente psiquiátrico pode gerar sobrecarga, porque constitui uma quebra no ciclo esperado de vida, que pressupõe que pessoas adultas sejam independentes.

Várias pesquisas internacionais ${ }^{(10)}$ são feitas desde a década de 1950 para avaliar o grau de sobrecarga gerada na família de um paciente psiquiátrico cujo cuidar causa sobrecarga. Estudos como esses vêm intensificando ainda mais que os cuidadores se sentem sobrecarregados.

Quando a mulher (esposa/mãe/dona de casa) é a portadora do transtorno mental, parece afetar muito mais a família, porque 
ela é o elo organizador do grupo ${ }^{(21)}$. Por intermédio dela ocorrem as transações e intermediações no grupo. Sua internação afeta intensamente todos os seus integrantes, "pois a ausência da mãe do âmbito doméstico" é o principal fator de desorganização dos grupos familiares dos seguimentos de baixa renda ${ }^{(22)}$.

Os resultados da presente pesquisa mostraram que os familiares precisavam assumir as despesas dos pacientes e que esses gastos correspondiam a uma boa parcela da renda familiar total.

A presença de sobrecarga tanto objetiva quanto subjetiva, obtida nos resultados dessa amostra, converge para a necessidade de os serviços de saúde incluírem ações para auxiliar os familiares cuidadores, ou seja, oferecer maiores esclarecimentos aos familiares sobre como lidar com os comportamentos problemáticos dos pacientes. Cabe ainda informar-lhes que a presença de alguns desses comportamentos pode indicar uma necessidade de reajuste na medicação dos pacientes, com consequente redução da sobrecarga familiar.

Em vista dos resultados, pode-se averiguar que os cuidadores que melhor tinham a aceitação do tratamento pelo paciente eram aqueles que conseguiam manter vínculos relacionais afetivos com ele.

A saúde mental não pode ser vista apenas como responsabilidade do poder público, mas também como responsabilidade da sociedade, com a mudança das condições de vida, na qualidade da mesma e dos serviços prestados aos usuários. Daí que o envolvimento das equipes de saúde com os familiares é capaz de promover a percepção de medos, dúvidas e curiosidades, trazendo a consequente diminuição da sobrecarga que lhes é atribuída.

\section{CONSIDERAÇÕES FINAIS}

A partir dessa pesquisa, podemos concluir que a sobrecarga do cuidador é grande, que ela o desvincula de seu próprio meio social em prol da atenção contínua ao paciente. Afeta e ainda quebra toda a evolução normal de um ser humano, que é se tornar um adulto independente.

Foi possível perceber também que a reforma psiquiátrica não é apenas papel do poder público, e sim de toda a sociedade, e principalmente daqueles que estão em contato direto e contínuo e possuem vínculos afetivos com um portador de transtorno mental crônico. É necessário reintegrá-lo ao convívio social para que ele mesmo, na medida do possível, possa ter alguma participação no curso de sua recuperação.

Não nos esqueçamos do fundamental papel da equipe de saúde na a promoção de esclarecimentos e ajuda tanto aos pacientes como aos seus cuidadores.

\section{Referências}

1. Gonçalves AM, Sena RRA. Reforma psiquiátrica no Brasil: contextualização e reflexos sobre o cuidado com o doente mental na família [Internet]. [citado em 2008 Dez 05. Disponível em: http://www.scielo.br/scielo.php?script=sci_arttext\&pi $d=S 010411692001000200007 \&$ Ing $=$ en\&nrm=iso

2. Schene AH. Objective and subjective dimensions of family burden: towards and integrative framework for research. Soc Psychiatry Epidemiol. 1990;25(6):289-97. 3. Lefley HP, Wasow M. (1994). Helping families cope with mental illness. Switzerland: H. P. Press

4. Lefley HP. Families, culture, and mental illness: constructing new realities. Psychiatr Interperson Biol Proc. 1985;61(4):335-55.

5. Campos PHF, Soares CB. Representação da sobrecarga familiar e adesão aos serviços alternativos em saúde mental [Internet]. [citado 2008 Dez 05]. Disponível em: http://pepsic.bvs-psi.org.br/scielo.php?script=sci_arttext\&pid=\$167711682005 000200006\&lng=pt\&nrm=iso

6. Tsu TMJA. A internação psiquiátrica e o drama das famílias. São Paulo: EDUSP; 1993. 7. Maurin JT, Boyd CB. Burden of mental illness on the family: a critical review. Arch Psychiatr Nurs. 1990;4(2):99-107.

8. Goldman HH. Mental illness and family burden: a public health perspective. J Am Psychiatr Assoc. 1982;33(7):557-60.

9. Sadigursky D. Desinstitucionalização do doente mental: expectativas da família [tese]. Ribeirão Preto: Escola de Enfermagem de Ribeirão Preto da USP; 1997. 10. Tessler RC, Gamache GM. Family experiences with mental illness. Westport: Auburn House; 2000.

11. Martens $L$, Addington J. The psychological well-being of family members of individuals with schizophrenia. Soc Psychiatry Psychiatr Epidemiol. 2001;36:128-33.
12. Hoenig J, Hamilton MW. The schizophrenic patient in the community and his effects on the household. Int J Soc Psychiatr. 1966;12:165-76.

13. Lauber C, Eichenberger A, Luginbühl P, Keller C, Rössler W. Determinants of burden in caregivers of patients with exacerbating schizophrenia. Eur Psychiatr. 2003;18:285-9.

14. Loukissa D. Family burden in chronic mental illness: a review of research studies. J Adv Nurs. 1995;21:248-55.

15. Rose LE. Families of psychiatric patients: a critical review and future research directions. ArchPsychiatr Nurs. 1996;10(2):67-76.

16. Bulger MW, Wandersman $A$, Goldman CR. Burdens and gratifications

of caregiving: appraisal parental care of adults with schizophrenia. Am J Orthopsychiatr. 1993;63:255-65.

17. Mcgilloway S, Donnelly M, Mays N. The experience of caring for former longstay psychiatric patients. Br J Clin Psychol. 1997;36:149-51.

18. Koga M. Convivência com a pessoa esquizofrênica: sobrecarga familiar [dissertação]. Ribeirão Preto: Universidade de São Paulo; 1997.

19. Magliano L, Fadden G, Madianos M, Caldas de Almeida JM, Held T, Guarneri M, et al. Burden on the families of patients with schizophrenia: results of the BIOMED I study. Soc Psychiatr Epidemiol. 1998;33:405-12.

20. Martínez A, Nadal S, Beperet M, Mendióroz P. Sobrecarga de los cuidadores familiares de pacientes con esquizofrenia: factores determinantes. Anales Sis San Navarra. 2000;23(1):101-10.

21. Rosa LCS. Transtorno mental e o cuidado na família. São Paulo: Cortez; 2003. 22. Zaluar A. A máquina e a revolta: as organizaçōes populares e o significado da pobreza. São Paulo: Brasiliense; 1985. 